To Appear in ApJ Letters

Preprint typeset using LATEX style emulateapj v. 04/03/99

\title{
EVOLUTION OF DUST EXTINCTION AND SUPERNOVA COSMOLOGY
}

\author{
TOMOnORI TOTANi ${ }^{1}$ And CHIAKI KoBAYASHI ${ }^{2}$ \\ ${ }^{1}$ National Astronomical Observatory, Mitaka, Tokyo 181-8588, Japan (E-mail: \\ totani@th.nao.ac.jp) \\ ${ }^{2}$ Department of Astronomy, School of Science, University of Tokyo, Bunkyo-ku, Tokyo \\ 113-0033, Japan; (E-mail: chiaki@astron.s.u-tokyo.ac.jp)
}

To Appear in ApJ Letters

\begin{abstract}
We have made a quantitative calculation for the systematic evolution of average extinction by interstellar dust in host galaxies of high-redshift Type Ia supernovae, by using a realistic model of photometric and chemical evolution of galaxies and supernova rate histories in various galaxy types. We find that average $B$ band extinction $\left\langle A_{B}\right\rangle$ at $z \sim 0.5$ is typically $0.1-0.2$ mag larger than present, under a natural assumption that dust optical depth is proportional to gas column density and gas metallicity. This systematic evolution causes average reddening with $E(B-V) \sim 0.025-0.05$ mag with the standard extinction curve, and this is comparable with the observational uncertainty of the reddening of high-redshift supernovae. Therefore, our result does not contradict the observations showing no significant reddening in high- $z$ supernovae. However, the difference in apparent magnitude between an open universe and a $\Lambda$-dominated flat universe is only $\sim 0.2 \mathrm{mag}$ at $z \sim 0.5$, and hence this systematic evolution of extinction should be taken into account in a reliable measurement of cosmological parameters. Considering this uncertainty, we show that it is difficult to discriminate between an open and $\Lambda$-dominated flat cosmologies from the current data.
\end{abstract}

Subject headings: cosmology: observations - dust, extinction — galaxies: evolution — supernovae: general

\section{INTRODUCTION}

Type Ia supernovae (SNe Ia) have been known as a representative standard candle in the universe, and used in measurements of cosmological parameters such as the Hubble constant $\left(H_{0}\right)$, density parameter $\left(\Omega_{M}\right)$, and the cosmological constant $\left(\Omega_{\Lambda}\right)$. Recently two independent groups have obtained the same result that a $\Lambda$-dominated flat universe is strongly favored, by using a few tens of $\mathrm{SNe}$ Ia at redshift $z \sim 0.5$ (Riess et al. 1998 [R98]; Perlmutter et al. 1999 [P99]). Extinction by dust could affect these analyses, and both groups have made a considerable effort to assess the systematic uncertainty due to extinction. Both groups reported that there is no significant color difference between the high- $z$ and local samples. In the sample of P99, average reddening $\langle E(B-V)\rangle$ is $0.033 \pm 0.014$ mag for the local sample and $0.035 \pm 0.022$ for the high$z$ sample (P99). The mean $B-V$ color of the high- $z$ R98 sample is $-0.13 \pm 0.05$ or $-0.07 \pm 0.05$ depending on two analysis methods, while expectation of unreddened color is -0.10 to -0.05 . However, there is a statistical uncertainty of $\gtrsim 0.03 \mathrm{mag}$ in the color difference, and in addition, there is a systematic uncertainty in the $\mathrm{K}$ correction of about $0.03 \mathrm{mag}$ (P99, and probably similar number also for R98). Therefore, there is observational uncertainty of at least $\sim 0.05 \mathrm{mag}$ in reddening evolution for both groups. Furthermore, these error estimates have been achieved by statistical averaging of many supernovae; typical color uncertainty for each supernova is much larger ( 0.1-0.2 mag, see Fig. 6 of P99). Therefore, it is difficult to clearly check a systematic evolution of average $B$-band extinction $\Delta A_{B} \sim R_{B} \Delta E(B-V) \sim 0.2$ mag with a reddening of $\Delta E(B-V) \sim 0.05$ by the current observational data, where $R_{B}$ is the total-to-selective extinction ratio for the $B$ band, and $R_{B} \sim 4$ for the standard extinction curve. On the other hand, the difference of apparent magnitude between an open universe and a $\Lambda$-dominated flat universe is only $\sim 0.2 \mathrm{mag}$ at $z \sim 0.5$, and hence it is still possible that unchecked systematic evolution of extinction has affected the measurements of cosmological parameters.

Aguirre (1999a, b) considered the effect of intergalactic dust which was ejected from galaxies. Such dust could have a significantly grey extinction and Aguirre has shown that this kind of dust may affect measurements of cosmological parameters with smaller reddening. This is an interesting possibility, but existence of such intergalactic dust has not yet been confirmed. Here we consider the dust existing in host galaxies with the standard extinction curve. Although it is uncertain whether supernovae evolve to high redshifts, their host galaxies should undoubtedly evolve as shown by various observations of galaxies at high redshifts. Both the gas column density and gas metallicity, which are important physical quantities for dust opacity, change with time by various star formation histories depending on morphological types of galaxies. Therefore, average dust extinction in host galaxies should evolve systematically, and the aim of this paper is to make a quantitative estimate for this evolution, by using a realistic model of photometric and chemical evolution of galaxies and supernova rate histories in various types of galaxies. 
We find that typical evolution in average $A_{B}$ is $\sim 0.1-0.2$ mag from $z=0$ to $\sim 0.5$, which is significant for measurements of cosmological parameters but may have escaped from the reddening check. Therefore, this effect should not be ignored in measurements of cosmological parameters by high- $z$ SNe Ia.

\section{EVOLUTION OF AVERAGE EXTINCTION IN HOST GALAXIES}

In this letter we consider only the average extinction of a supernova in a host galaxy, and do not consider the variation within a galaxy depending on the supernova location in it. Although the variation within a host galaxy can be washed out by statistical averaging of many supernovae, evolution of galaxies will cause systematic evolution of average extinction which cannot be removed by statistical averaging. It is physically natural to assume that the dust-yo-netal ratio is constant and hence the tust opacity

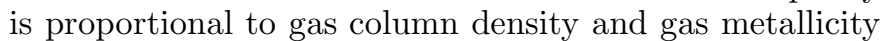
of a host galaxy. In fact, it is well known that the extinction in Galaxy is density (e.g., Burstein \& Heiles 1982; Pei 1992). It is also known that the dust opacity is correlated to the metallicity among the Galaxy and the Large and Small Magellanic Clouds, when gas column density is fixed (e.g., Pei 1992). Hence in the following we assume that the dust opacity is proportional to gas column density and gas metallicity, which evolve according to the star formation history in a galaxy.

The star formation history can be inferred from the present-day properties of observed galaxies, by using the well-known technique of stellar population synthesis. We can estimate the time evolution of gas fraction and metallicity in a galaxy by using photometric and chemical evolution models for various galaxy types. Since the observed extinction of high- $z$ SNe Ia is an average over various types of galaxies, we also need the evolution of SN Ia rate in various galaxy types. In the next section, we describe the model of galaxy evolution and SN Ia rate evolution used in this letter, which is constructed to reproduce various properties of the present-day galaxies.

\subsection{Evolution of galaxies and Type Ia supernova rate}

We use photometric and chemical evolution models for five morphological types of E/S0, S0a-Sa, Sab-Sb, Sbc-Sc, and Scd-Sd. The basic framework of the model is the same as that of elliptical galaxies of Arimoto \& Yoshii (1987) and that of spiral galaxies of Arimoto, Yoshii, \& Takahara (1992), but model parameters are updated to match the latest observations (Kobayashi et al. 1999), by using an updated stellar population database of Kodama \& Arimoto (1997) and nucleosynthesis yields of supernovae of Tsujimoto et al. (1995). The model parameters for spiral galaxies are determined to reproduce the present-day gas fractions and $B-V$ colors in various galaxy types at 15 Gyr after the formation. The model of elliptical galaxies is the so-called galactic wind model, in which star formation stops at about 1 Gyr after the formation by a supernova-driven galactic wind (Larson 1974; Arimoto \& Yoshii 1987). We assume that gas fraction in a elliptical galaxy decreases exponentially after the galactic wind time ( $\sim 1 \mathrm{Gyr})$, with a time scale same as the galactic wind time. These models give the evolution of gas fraction and metallicity in each galaxy type depending on the star formation history.

SN Ia rate history in each type of galaxies is calculated with the metallicity-dependent SN Ia model introduced by Kobayashi et al. (1998). In their SN Ia progenitor model, an accreting white dwarf (WD) blows a strong wind to reach the Chandrasekhar mass limit. If the iron abundance of progenitors is as low as $[\mathrm{Fe} / \mathrm{H}] \lesssim-1$, the wind is too weak for SNe Ia to occur. Their SN Ia scenario has two progenitor systems: one is a red-giant (RG) companion with the initial mass of $M_{\mathrm{RG}, 0} \sim 1 M_{\odot}$ and an orbital period of tens to hundreds days (Hachisu, Kato, \& Nomoto 1996, 1999). The other is a near main-sequence (MS) companion with an initial mass of $M_{\mathrm{MS}, 0} \sim 2-3 M_{\odot}$ and a period of several tenths of a day to several days (Li \& van den Heuvel 1997; Hachisu et al. 1999). The occurrence of SNe Ia is determined from two factors: lifetime of companions (i.e., mass of companions) and iron abundance of progenitors. (See Kobayashi et al. 1998, 1999 for detail.) This model successfully reproduces the observed chemical evolution in the solar neighborhood such as the evolution of oxygen to iron ratio and the abundance distribution function of disk stars (Kobayashi et al. 1998), the present SN II and Ia rates in spirals and ellipticals, and cosmic SN Ia rate at $z \sim 0.5$ (Kobayashi et al. 1999).

\subsection{Average extinction evolution towards high redshifts}

We have modeled the evolution of gas fraction $\left(f_{\mathrm{g}}\right)$, metallicity $(Z)$, and SN Ia rate per unit baryon mass of a galaxy $\left(\mathcal{R}_{I a}\right)$ in various types of galaxies, from which we calculate the evolution of average extinction in the universe. We assume that these quantities do not depend on the mass of galaxies. The basic assumption is that the dust opacity, and hence average $A_{B}$ in a galaxy is proportional to gas column density and gas metallicity. The average extinction at redshift $z$ in a $i$-th type galaxy with the present-day $B$ luminosity $L_{B}$ is given by $A_{B, i}\left(z, L_{B}\right)=\kappa f_{\mathrm{g}, i}\left(t_{z}\right) Z_{i}\left(t_{z}\right)\left[r_{\mathrm{e}, i}\left(L_{B}\right)\right]^{-2}\left(M_{\mathrm{b}} / L_{B}\right)_{i} L_{B}$, where $t_{z}$ is the time from formation of galaxies, $r_{\mathrm{e}}$ the effective radius of galaxies, and $\left(M_{\mathrm{b}} / L_{B}\right)$ is the baryon-mass to light ratio which is determined by the evolution model. We assume a single formation epoch $z_{F}$ for all galaxy types for simplicity. ${ }^{1}$ The proportional constant $\kappa$ will be determined later. We do not consider the size evolution of galaxies, and determine $r_{\mathrm{e}}\left(L_{B}\right)$ from empirical relations observed in local galaxies (Bender et al. 1992 for ellipticals, and Mao \& Mo 1998 for disk galaxies). It should be noted that the extinction depends on the absolute luminosity of galaxies. From the empirical $L_{B}-r_{\mathrm{e}}$ relation, the surface brightness becomes brighter with increasing luminosity of disk galaxies, and hence the massive galaxies

${ }^{1}$ In reality, there should be some dispersion in galaxy ages. However, the systematic evolution of dust extinction is owing to the fact that all galaxies should become younger on average towards high redshifts, and present-day age dispersion cannot remove this systematic effect. 
should be more dusty than smaller ones. This trend is consistent with observations (van den Bergh and Pierce 1990; Wang 1991). Then the average extinction of SNe Ia over all galaxy types at a given redshift is

$$
\left\langle A_{B}(z)\right\rangle=\frac{\sum_{i} \int d L_{B} A_{B, i}\left(z, L_{B}\right) \mathcal{R}_{\mathrm{Ia}, i}\left(t_{z}\right)\left(M_{\mathrm{b}} / L_{B}\right)_{i} L_{B} \phi_{i}\left(L_{B}\right)}{\sum_{i} \int d L_{B} \mathcal{R}_{\mathrm{Ia}, i}\left(t_{z}\right)\left(M_{\mathrm{b}} / L_{B}\right)_{i} L_{B} \phi_{i}\left(L_{B}\right)}
$$

where $\phi_{i}$ is the type-dependent galaxy luminosity function at $z=0$, for which we adopted the Schechter parameters derived by Efstathiou, Ellis, \& Peterson (1988) using the catalog of the Center for Astrophysics (CfA) Redshift Survey (Huchra et al. 1983).

We have to determine the overall normalization of extinction, $\kappa$, for which we use the average $V$ extinction of the Milky Way (Sbc type, $L_{B}=1.4 \times 10^{10} L_{B \odot}$ ) at $z=0$ : $\left\langle A_{V}\right\rangle_{\mathrm{MW}}$. This is an average of extinction of SNe Ia occurring in our Galaxy seen by an extragalactic observer, and hence it is different, in a strict sense, from the average of the Galactic extinction which is extinction of extragalactic objects observed by us. However, if the location of the Sun is typical in the Milky Way, we may infer this quantity by the average of the Galactic extinction. The average Galactic extinction of the $42 \mathrm{SNe}$ Ia observed by P99 is $\sim 0.1 \mathrm{mag}$ in $A_{R}$ or $A_{I}$ (see Table 1 of P99). This suggests $\left\langle A_{V}\right\rangle_{\mathrm{MW}} \sim 0.1-0.2$ with the standard Galactic extinction law (e.g., Pei 1992). The average reddening of the Galaxy then becomes $\langle E(B-V)\rangle_{\mathrm{MW}} \sim 0.03-0.06$ $\mathrm{mag}$, which is a typical reddening at the Galactic latitude of $\sim 40-50^{\circ}$ in the Galactic extinction map (Burstein \& Heiles 1982; Schlegel, Finkbeiner, \& Davis 1998). This estimate is consistent with a model of dust distribution in our Galaxy, which suggests that the average extinction of $\mathrm{SNe}$ Ia in the Galaxy seen by an extragalactic observer is typically $\left\langle A_{V}\right\rangle_{\mathrm{MW}} \sim 0.1-0.2 \mathrm{mag}$ (Hatano, Branch, $\&$ Deaton 1998). ${ }^{2}$ Therefore we use $\left\langle A_{V}\right\rangle_{\mathrm{MW}} \sim 0.1-0.2$ mag as a plausible range of the average extinction of our Galaxy.

Figure 1 shows the evolution of $B$ extinction for each galaxy type as well as the average over all galaxy types, normalized by $\left\langle A_{V}\right\rangle_{\mathrm{MW}}$, i.e., $\left\langle A_{B}(z)\right\rangle /\left\langle A_{V}\right\rangle_{\mathrm{MW}}$. Here we used a cosmological model with $\left(h, \Omega_{M}, \Omega_{\Lambda}\right)=(0.5,0.2,0)$, and set $z_{F}=4.5$ so that the age of galaxies is 15 Gyr which was assumed in the evolution model. The thick solid line is the average over all galaxy types, and the thin lines are for individual galaxy types as indicated. Since we have used a galactic wind model for elliptical galaxies, they do not have interstellar gas and hence there is no extinction in elliptical galaxies at $z<1$. The evolution of extinction is caused by spiral galaxies, but the behavior of evolution is considerably dependent on galaxy types. Early-type spiral galaxies become more dusty towards $z \sim 1$, but an opposite trend is seen for late types. These behaviors can be understood as a competition of the two effects: gas fraction evolution and metallicity evolution. The gas fraction in early spiral galaxies is much smaller than late types at present, but rapidly increases towards high redshifts. This increase is responsible for increase of gas column density and hence the dust opacity. On the other hand, the gas fraction does not increase so much in late type galaxies, and decrease of metallicity towards high redshifts is responsible for the decrease of dust opacity. In redshifts more than 1, the extinction decreases towards higher redshifts in all spiral galaxies because the metallicity evolution becomes dominant.

The average over all types is weighted by the SNe Ia rate in each type. Because the star formation rate increases more rapidly to high redshifts in early-type spiral galaxies than late types, the average extinction is more weighted to early types at higher redshifts. Hence $\left\langle A_{B}\right\rangle /\left\langle A_{V}\right\rangle_{\mathrm{MW}}$ increases to high redshifts by $\sim 1$ from $z=0$ to 0.5 . This result suggests that, with $\left\langle A_{V}\right\rangle_{\mathrm{MW}} \sim 0.1-0.2$, the average extinction $\left\langle A_{B}\right\rangle$ of SNe Ia at $z \sim 0.5$ is larger than the local sample by about $0.1-0.2$ mag. This systematic evolution of average extinction is comparable with the difference between an open and a $\Lambda$-dominated universe in the Hubble diagram, and hence this effect significantly affects measurements of cosmological parameters. In the next section we apply the above model in the estimate of cosmological parameters by using the sample of P99.

\section{EFFECT ON THE COSMOLOGICAL PARAMETERS}

Figure 2 shows the Hubble diagram for $\mathrm{SNe}$ Ia of the primary fit $\mathrm{C}$ of $\mathrm{P} 99$, which plots restframe $B$ magnitude residuals from a $\Lambda$-dominated flat cosmology $\left[\left(h, \Omega_{M}, \Omega_{\Lambda}\right)=(0.65,0.2,0.8)\right]$ without dust effect (thin solid line). Thin long- and short-dashed lines are the predictions of the dust-free case with an open universe $(0.5,0.2,0.0)$ and the Einstein-de Sitter (EdS) universe $(0.5,1.0,0.0)$, respectively. As reported by P99, the $\Lambda$-dominated flat universe gives the best-fit to the data. Next, the thick lines show the predictions when the model of extinction evolution is taken into account, where the cosmological parameters are the same with the dust-free curves of the same line-markings. Here we adopt $\left\langle A_{V}\right\rangle_{\mathrm{MW}}$ $=0.2 \mathrm{mag}$. In the open and $\Lambda$-dominated models, the galaxy formation epoch is set to $z_{F}=4.5$ and 5.0 so that the age of galaxies becomes 15 Gyr. In the EdS model, the age of the universe is shorter than $15 \mathrm{Gyr}$, and hence we set $z_{F}=5$ which gives an age of galaxies as $\sim 12$ Gyr. Although this age is a little shorter than that assumed in the evolution model, the evolutionary effect during 12-15 Gyr is small and hence this inconsistency is not serious. As expected, the model curves with the dust effect are typically $0.1-0.2 \mathrm{mag}$ fainter than those without dust. As a result, the open universe becomes the most favored cosmology among the three when the extinction evolution is taken into account.

We avoid more detailed statistical analysis to derive any decisive conclusion about the cosmological parameters, because the result would be highly dependent on the extinction evolution model. However, the evolution model presented here is quite a natural and standard one without any exotic assumption. Hence, our conclusion is that the systematic evolution of average extinction in host galaxies

${ }^{2}$ Hatano et al. suggested that most supernovae are only mildly obscured but there is a long tail to stronger extinctions in the extinction distribution. In the actual observations, such a tail will be cut out due to a magnitude limit of a survey. Hence, we have used here the mean extinction of the "extinction-limited subset" in the Table 1 of Hatano et al., in which strongly obscured supernovae with $A_{B}>0.6$ are removed. 
should more carefully be taken into account when one uses SNe Ia to constrain the cosmological parameters.

\section{DISCUSSION}

P99 estimated the systematic uncertainty of extinction to be less than $0.025 \mathrm{mag}(1 \sigma)$ in $A_{B}$, based on an analysis after removing nine reddest supernovae. Aguirre (1999b) argued that this limit does not apply if the dispersion in brightness and/or colors of high- $z$ supernovae is dominated by factors other than extinction. As noted before, the observational uncertainty in $E(B-V)$ for each supernova is typically $\sim 0.1-0.2 \mathrm{mag}$, which is larger than the systematic reddening of $E(B-V) \sim 0.025-0.05$ considered in this letter. Therefore, it is doubtful that P99 analysis successfully removed high- $z$ supernovae reddened by the systematic extinction evolution. Rather, the supernovae removed by P99 might be reddened by strong reddening depending on locations in host galaxies, or by some other reasons as argued by Aguirre (1999b). Hence, we consider that the systematic evolution of dust considered here has not yet been checked by the observations.

In the analysis of R98, the reddening correction is systematically included in the process of light-curve-shape fitting, and one may consider that our result does not apply to the analysis of R98. However, as noted in Introduction, one must correct a reddening with $E(B-V) \sim 0.05$ to discriminate between an open and $\Lambda$ cosmologies, and this is comparable with the observational error of color estimates. In principle, it is difficult to correct the reddening effect when the reddening is as small as the observational error in colors, because the reddening correction is performed based on the observed colors. The reddening correction by R98 will be effective for supernovae strongly reddened beyond the color uncertainty, but it is not clear to us whether this correction has successfully corrected the systematic reddening evolution discussed in this letter.
The two groups (R98, P99) argued that the observed dispersion of apparent magnitudes showing no significant evolution to high redshifts gives further support that their results are not affected by extinction. Their argument on the dispersion test is true if the observed dispersion is dominated by the dispersion of extinction, and R98 claimed that the observed dispersion is smaller than that expected from a dust distribution model of Hatano et al. (1998) if mean extinction is $\sim 0.2 \mathrm{mag}$. However, there is large uncertainty in dust distribution models, and the selection effect might have reduced the apparent dispersion (see the footnote 2). We should also be careful about the uncertainty in the observed 'intrinsic' dispersion, which was derived by subtracting the measurement error from the actually observed dispersion (P99). Hence, this test does not favor our scenario but cannot strictly reject it.

We suggest that the best way to constrain the cosmological parameters by high- $z$ SNe Ia would be an analysis using only SNe Ia in elliptical galaxies, in which the dust evolution effect is much smaller than spiral galaxies at $z<1$. In fact, P99 tried to analyze their supernovae with known host-galaxy types, and found no significant change in the best-fit cosmology. However, the host-galaxy classification is only based on spectra of host galaxies without highresolution images. The uncertainty of a fit with a specified host-galaxy type is still large due to the limited number of supernovae, and P99 concluded that this test will need to await the host-galaxy classification of the full set of high- $z$ supernovae and a larger low- $z$ supernova sample. It is expected from our calculation that the fitting result of such an analysis in the future will be dependent on host-galaxy types, giving important information for chemical evolution of galaxies. High- $z$ supernovae beyond $z \sim 1$ are also desirable to study galaxy evolution as well as cosmological parameters.

\section{REFERENCES}

Aguirre, A.N., 1999a, ApJ, 512, L19

Aguirre, A.N., 1999b, ApJ, in press astro-ph/9904319

Arimoto, N., \& Yoshii, Y. 1987, A\& A, 173, 23

Arimoto, N., Yoshii, Y., \& Takahara, F., 1992, A\&A, 253, 21

Bender, R., Burstein, D. \& Faber, S.M. 1992, ApJ, 399, 462

Burstein, D. \& Heiles, C. 1982, AJ, 87, 1165

Efstathiou, G., Ellis, R.S., \& Peterson, B.A. 1988, MNRAS, 232, 431

Hachisu, I., Kato, M., \& Nomoto, K. 1996, ApJ, 470, L97

Hachisu, I., Kato, M.. \& Nomoto, K. 1999, ApJ, 521, in press astro-ph/9902304

Hachisu, 1., Kato, M., Nomoto, K., \& Umeda, H. 1999, ApJ, 519, in press astro-ph/9902303

Hatano, K., Branch, D., \& Deaton, J. 1998, ApJ, 502, 177

Huchra, J.P., Davis, M., Latham, D. \& Tonry, J. 1983, ApJS, 52, 89
Kobayashi, C., Tsujimoto, T., Nomoto, K., Hachisu, I, \& Kato, M. 1998, ApJ, 503, L155

Kobayashi, C., Tsujimoto, T. \& Nomoto, K. 1999, submitted to ApJ, astro-ph/9908005

Kodama, T., \& Arimoto, N.. 1997, A\&A, 320, 41

Larson, R. B., 1974, MNRAS, 169, 229

Li, X. -D., \& van den Heuvel, E. P. J. 1997, A\&A, 322, L9

Mao, S. \& Mo, H.J. 1998, MNRAS 296, 847

Pei, Y.C. 1992, ApJ, 395, 130

Perlmutter, S. et al. 1999, ApJ, 517, 565 (P99)

Riess, A.G. et al. 1998, AJ, 116, 1009 (R98)

Schlegel, D., Finkbeiner, D., \& Davis, M. 1998, ApJ, 500, 525

Tsujimoto, T., Nomoto, K., Yoshii, Y., Hashimoto, M.,

Yanagida, S., \& Thielemann, F.-K. 1995, MNRAS, 277, 945

van den Bergh, S. \& Pierce, M.J. 1990, ApJ, 364, 444

Wang, B. 1991, ApJ, 383, L37 


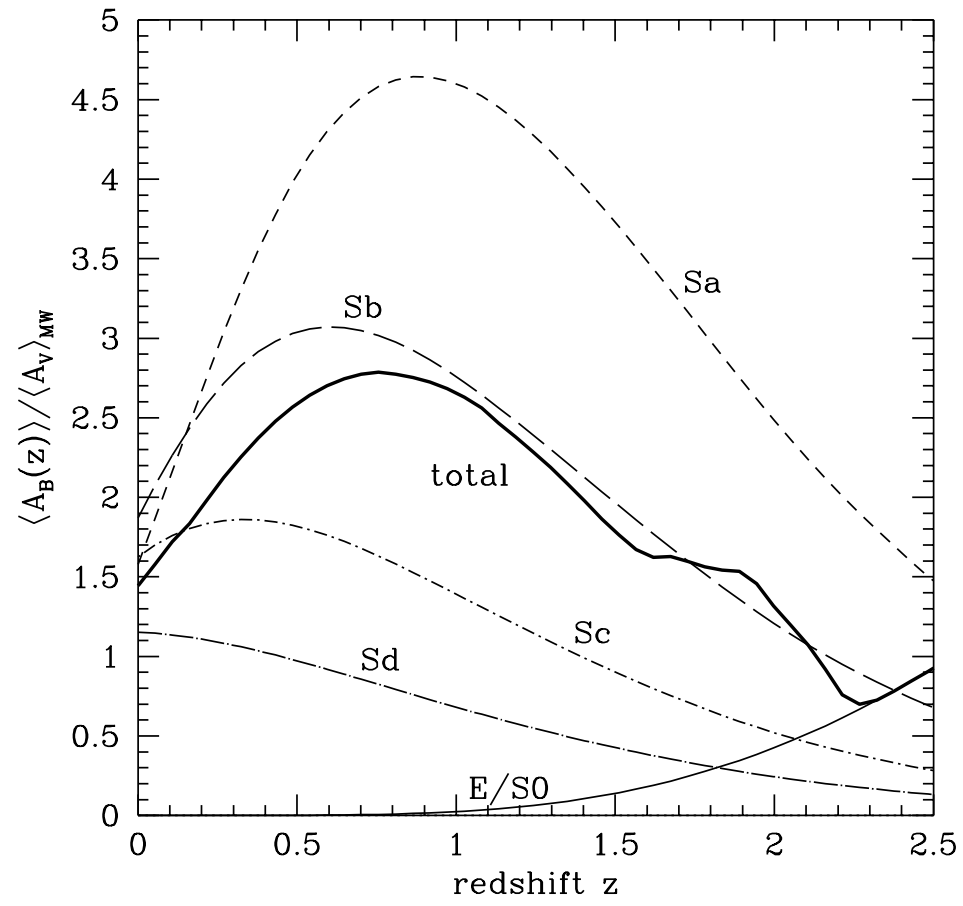

Fig. 1.- Average $B$-band extinction of type Ia supernovae as a function of redshift. Extinction $A_{B}$ is normalized by the average $V$ extinction of our present-day Galaxy, $\left\langle A_{V}\right\rangle_{\mathrm{MW}}$ (see text). The thick solid line is the average over the five morphological types of galaxies, which is weighted by SN Ia rate in them. Five thin lines are extinction evolution in individual galaxy types, as indicated. An open universe with $\left(h, \Omega_{M}, \Omega_{\Lambda}\right)=(0.5,0.2,0.0)$ is assumed, and the formation epoch of galaxies is set to $z_{F}=4.5$. 


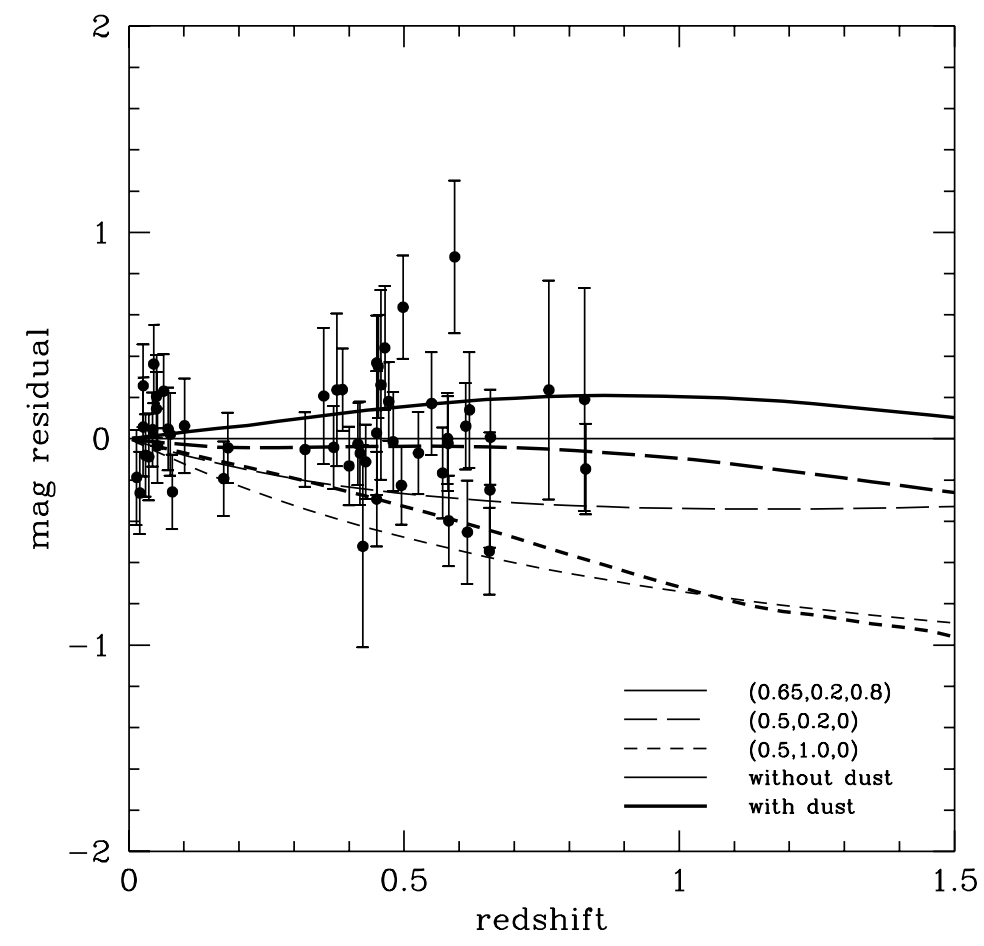

Fig. 2. - The Hubble diagram of type Ia supernovae. The data are those used in the primary fit $\mathrm{C}$ of Perlmutter et al. (1999). Restframe $B$ Magnitude residuals are from a $\Lambda$-dominated universe with $\left(h, \Omega_{M}, \Omega_{\Lambda}\right)=(0.65,0.2,0.8)$ without dust effect (thin solid line). Thin long- and short-dashed lines are predictions of an open universe $(0.5,0.2,0.0)$ and Einstein-de Sitter universe $(0.5,1.0,0.0)$ without dust. The three thick lines are predictions for the case with dust evolution, where the cosmological parameters are the same with the dust-free curves of the same line-markings. 